\title{
Expression of circular RNA CDR1-AS in colon cancer cells increases cell surface PD-L1 protein levels
}

\author{
ERI TANAKA, YU MIYAKAWA, TAKAHIRO KISHIKAWA, TAKAHIRO SEIMIYA, \\ TAKUMA IWATA, KAZUYOSHI FUNATO, NARIAKI ODAWARA, \\ KAZUMA SEKIBA, MARI YAMAGAMI, TATSUNORI SUZUKI, \\ REI ISHIBASHI, MOTOYUKI OTSUKA and KAZUHIKO KOIKE \\ Department of Gastroenterology, Graduate School of Medicine, \\ The University of Tokyo, Tokyo 113-8655, Japan
}

Received February 10, 2019; Accepted June 7, 2019

DOI: $10.3892 /$ or.2019.7244

\begin{abstract}
The expression of CDR1-AS, a representative circular RNA, is closely linked with poor prognosis in gastrointestinal cancers, such as colon, liver, and pancreatic cancers. Although it is well known that CDR1-AS antagonizes microRNA-7 function through its sequence similarities in the brain, its biological function and link with the malignant potential of cancer cells remain unclear, partly due to the difficulties of ectopic expression of circular RNAs. In the present study, SW620, a colon cancer cell line that stably expresses CDR1-AS RNA circularized, was established using the laccase 2 gene cassette, and its biological function associated with malignant behavior was determined. In contrast to previous studies, cell growth or invasion ability was not altered by CDR1-AS expression. However, the expression levels of CMTM4 and CMTM6, which were recently recognized as critical regulators of PD-L1 protein expression at the cell surface, were significantly increased. Accordingly, the cell surface PD-L1 protein levels were increased in CDR1-AS-expressing cells. Notably, the effects were not canceled out by overexpressing microRNA-7, indicating that the increase in cell surface PD-L1 in CDR1-AS-expressing cells was not dependent on microRNA-7 function. These results indicated that expression of this circular RNA in cancer cells may lead to poor prognosis by increasing cell surface PD-L1 levels through microRNA-7-independent mechanisms.
\end{abstract}

Correspondence to: Dr Motoyuki Otsuka, Department of Gastroenterology, Graduate School of Medicine, The University of Tokyo, 7-3-1 Hongo, Bunkyo-ku, Tokyo 113-8655, Japan

E-mail: otsukamo-tky@umin.ac.jp

Abbreviations: circRNAs, circular RNAs; CDR1-AS, cerebellar degeneration-related protein 1 transcript; miR, microRNA

Key words: circular RNA, colon cancer, PD-L1, CDR1-AS, CMTM

\section{Introduction}

Circular RNAs (circRNAs) are a novel class of endogenous noncoding RNAs. circRNAs were long thought to be artifacts of aberrant RNA splicing, however, the recent development of next-generation sequencing has led to the discovery of numerous endogenous circRNAs with unique functions. In fact, circRNAs are functionally involved in normal processes as well as pathogenic status (1-3).

Colorectal cancer is a leading cause of tumor-associated mortality worldwide, and its incidence continues to gradually increase (4). Recently, it was reported that a representative circRNA, cerebellar degeneration-related protein 1 transcript (CDR1-AS), is frequently upregulated in colorectal cancer (5). Since CDR1-AS can bind $>70$ copies of microRNA-7 (miR7) due to their sequence similarities, CDR1-AS functions as a competing endogenous RNA (ceRNA) against miR7 $(6,7)$. miR7 can function as either an oncogenic or tumor-suppressive microRNA depending on the cellular context, and may exert tumor-suppressive effects in colorectal cancer (5). In fact, colon cancer cases with CDR1-AS expression exhibited a poorer prognosis, and overexpression of CDR1-AS in colon cancer cells resulted in biological consequences due to functional impairment of miR7 when it was overexpressed (5). However, because miR7, which is principally expressed in neuroendocrine cells (8), is not always abundant in colon tissues, it was hypothesized that CDR1-AS may have functions other than the impairment of miR7 that lead to worse prognosis in colorectal carcinoma.

In the present study, to examine CDR1-AS function in colorectal cancer cells, the factors that potentially influence colorectal cancer prognosis due to the overexpression of CDR1-AS were determined. The present results may provide insights into novel mechanisms underlying the link between CDR1-AS expression and poor prognosis in colorectal cancer.

\section{Materials and methods}

Cells. The cell line 293T and the human colon carcinoma cell lines SW620 and Caco2 were purchased from the American Type Culture Collection and cultured in Dulbecco's Modified 
Eagle's medium (DMEM) or L-15 medium (Leibovitz), respectively, supplemented with $10 \%$ fetal bovine serum. Human umbilical vein endothelial cells (HUVECs) were purchased from PromoCell and cultured in endothelial cell Growth Medium 2 (PromoCell).

Plasmid production and lentivirus infection. The CDR1-AS-expressing plasmid,pcDNA3.1-laccase2-MCS-ciRS7, whose transcript naturally circularizes due to DNAREPI_DM cassettes, and its control plasmid were obtained from Addgene, Inc. The lentiviral vector pCDH-CDR1-AS was constructed by subcloning the corresponding sequences from pcDNA3.1 into pCDH vector, using the EcoRI and NotI sites. Lentiviruses produced from the pCDH control vector were used as a negative control.

To generate stably expressed polyclonal cells, the Lentivirus Packaging System (System Biosciences) was used according to the manufacturer's protocol. The viruses were transduced into SW620 cells and Caco2 cells using polybrene (Santa Cruz Biotechnology, Inc.), followed by selection with $6 \mu \mathrm{g} / \mathrm{ml}$ puromycin to obtain polyclonal cells stably expressing CDR1-AS RNA.

RNA extraction and RNase $R$ treatment. RNA was extracted using ISOGEN II (Nippon Gene Co., Ltd.) according to the manufacturer's protocol. After RNA extraction, $6 \mu \mathrm{g}$ RNA was treated with RNase R (AR Brown Co., Ltd.) at $37^{\circ} \mathrm{C}$ for $15 \mathrm{~min}$, followed by purification using the RNeasy MinElute Cleanup kit, according to the manufacturer's protocol (Qiagen).

Northern blotting. Northern blotting was performed as described previously (9). Briefly, $5 \mu \mathrm{g}$ of RNAs were separated in $1 \%$ formaldehyde-denatured agarose gel and hydrostatically transferred to a Hybond $\mathrm{N}^{+}$membrane (GE Healthcare Life Sciences). Membranes were UV-crosslinked and hybridization was performed overnight at $42^{\circ} \mathrm{C}$ in ULTRAhyb Buffer (Ambion; Thermo Fisher Scientific, Inc.) containing $10 \mathrm{ng} / \mathrm{ml}$ biotin-labeled RNA probe. The membranes were stringently washed and bound probe was visualized using a BrightStar BioDetect kit (Ambion) according to the manufacturer's protocol. The probes for detecting CDR1-AS were generated using in vitro transcription MEGAscript T7 kit, and the pre-made probe for $\beta$-actin was obtained using a DIG Northern Starter kit (Roche Diagnostics).

Cell growth, hanging-drop method, and cell invasion. The Cell Counting Kit-8 (CCK-8; Dojindo Molecular Technologies, Inc.) was used to evaluate $2 \mathrm{D}$ cell growth according to the manufacturer's instructions. Cells were seeded at $1 \times 10^{3}$ cells/well in 96-well plates. CCK-8 solution was added at 24-, 48-, 72-, and 96-h time-points. The absorbance at $450 \mathrm{~nm}$ was measured using a microplate reader (Thermo Fisher Scientific, Inc.).

For the 3D cell culture, SW620 cells $\left(5 \times 10^{4}\right.$ cells/well) were seeded in hanging droplets in a volume of $40 \mu \mathrm{l}$ medium/droplet using the GravityPlus system (InSphero AG). After 8 days, the spheroids were transferred to a 96-well microtissue receiver plate with a non-adhesive surface (GravityTRAP; InSphero) in a volume of $70 \mu \mathrm{l}$ medium/well. CellTiter-Glo 3D cell reagent (Promega Corp.) was added at 24-, 48-, 72-, and 96-h time-points. Following $30 \mathrm{~min}$ of incubation at room temperature, luminescence was quantified on a GloMax 96 Microplate Luminometer (Promega Corp.).

An invasion assay was performed using a CytoSelect 96-well cell invasion assay kit (Cell Biolabs, Inc.) with $1 \times 10^{5}$ cells placed in the upper chamber of the assay plate. Chemoattractant was added to the feeder tray. The assay plate was cultured for $24 \mathrm{~h}$, and cells that migrated were collected and quantified using CyQUANT GR dye solution (Cell Biolabs, Inc.). Invasion values were reported as the mean relative fluorescence units (RFUs) measured at $480 \mathrm{~nm} / 520 \mathrm{~nm}$ using a GloMax Discover Multimode Microplate Reader (Promega Corp.).

Angiogenesis assay. An Angiogenesis Assay Kit (PromoCell) was used to evaluate endothelial tube formation according to the manufacturer's instructions. HUVECs were seeded onto 96-well plates coated with basement membrane extract at $1.0 \times 10^{4}$ cells/well. The culture supernatant of the negative control and the CDR1-AS-expressing SW620 cells were added to each well, and the plate was incubated for $6.5 \mathrm{~h}$. The tubular networks were analyzed using Image J software $1.51 \mathrm{~J} 8$ (National Institutes of Health).

cDNA microarray analyses. cDNA microarray analysis was performed using cDNA oligo chips (Toray Industries, Inc.). The data and the protocols were deposited in a public database (GEO; accession no. GSE125687).

$R T-q P C R$. Quantitative RT-PCR (qRT-PCR) was performed as described previously (10). All values were normalized to the mRNA levels of the $\beta$-actin. Relative expression was calculated according to the $\Delta \Delta \mathrm{C}_{\mathrm{q}}$ method as follows: $\Delta \Delta \mathrm{C}_{\mathrm{q}}=\Delta \mathrm{C}_{\mathrm{q} \text { sample }}$ $-\Delta \mathrm{C}_{\mathrm{q} \beta \text {-actin }}$ (11). The primers used were as follows: CDR1-AS forward, 5'-GCTGATCTTCTGACATTCAGG-3' and reverse, 5'-GAGTTGTTGGAAGACCTTGAC-3'; PD-L1 forward, 5'-GGTGCCGACTACAAGCGAAT-3' and reverse, 5'-AGC CCTCAGCCTGACATGTC-3'; CMTM4 forward, 5'-CTG GCGTCTTGCTGATTATG-3' and reverse, 5'-ATTTCTGCT CCGGCTCTATG-3'; CMTM6 forward, 5'-ATGAAGGCC AGCAGAGACAG-3' and reverse, 5'-GTGTACAGCCCCACT ACGGA-3'; $\beta$-actin forward, 5'-TCCCTGGAGAAGAGCTAC GA-3' and reverse, 5'-AGCACTGTGTTGGCGTACAG-3'.

Transfection, reporter assay, and miR mimic. pGL4-miR7RE, a luciferase-based reporter construct to monitor miR7 function, was generated by inserting two tandem sequences of the miR7 responsive elements (ACAACAAAATCACTAGTCTTCCAA CAACAAAATCACTAGTCTTCCA) into the FseI site at the 3'UTR of the pGL4.5 luciferase vector (Promega Corp.). To construct the control mutated miR7 reporter, the following insertion sequence was used: ACAACAAAATCACTAGTC AAGGTACAACAAAATCACTAGTCAAGGT, which has mutations in the seed sequences of the miR7-recognizing sites.

To determine the functional activities of miR7, the pGL4-miR7RE plasmid, along with a pGL4-TK plasmid-expressing sea pansy luciferase (Promega Corp.) as an internal control, were transiently transfected into the cells using FuGENE6 (Promega Corp.). When adding the miR7 mimic, locked nucleic acid (LNA)-based miR7 oligonucleotides (Qiagen) were transfected using oligofectamine 
(Invitrogen; Thermo Fisher Scientific, Inc.) at the same time as the reporter transfection. At $48 \mathrm{~h}$ after transfection, dual luciferase assays were performed using a Dual-Luciferase Reporter Assay System (Promega Corp.) as previously described (10).

Flow cytometry. Flow cytometric analyses were performed as previously described (12). Cells were hybridized with anti-PDL1 (1:1,000; cat. no. PA5-20343; Thermo Fisher Scientific, Inc.) or the isotype control IgG (1:1,000; cat. no. 2729; Cell Signaling Technology, Inc.) for $40 \mathrm{~min}$ at $4^{\circ} \mathrm{C}$. After washing, the cells were incubated with goat anti-mouse Alexa Fluor 488 (1:1,000; Molecular Probes; Thermo Fisher Scientific, Inc.) for $20 \mathrm{~min}$. Flow cytometry was performed, and the data were analyzed using Guava Easy Cyte Plus (GE Healthcare Life Sciences). To quantify the PD-L1-positive cells, cells with fluorescence intensity exceeding the negative control cells were counted, and the ratio was calculated.

Mice and xenograft. Experimental protocols were approved by the Ethics Committee for Animal Experimentation at the University of Tokyo (approval no. P18-017) and conducted in accordance with the Guidelines for the Care and Use of Laboratory Animals of the Department of Medicine, University of Tokyo.

Four 8-week old male BALB/c (nu/nu) nude mice (weight $\sim 30 \mathrm{~g}$ ) were purchased from CREA Japan and maintained in 14/10-h light/dark cycle at $25^{\circ} \mathrm{C}$. Briefly, $2 \times 10^{6}$ control or CDR1-AS cells were suspended in $30 \mu \mathrm{l}$ of PBS containing $1 \%$ Matrigel (BD Biosciences) and injected subcutaneously into two mice to establish xenograft models. Mice were placed in standard conditions (4/cage) under specific pathogen-free conditions in laminar flow cabinets. All animals received food and water ad libitum. At 4 weeks post-transplantation, xenograft tissues were collected for immunohistochemistry analyses.

Immunohistochemistry. Immunohistochemistry was performed as previously described (13). Tissues were incubated overnight at $4^{\circ} \mathrm{C}$ with anti-PDL1 antibody (cat. no. PA5-20343; Thermo Fisher Scientific, Inc.) diluted with Can Get Signal Immunostain Immunoreaction Enhancer Solution (Toyobo Life Science). Signals were enhanced using a VECTASTAIN ABC kit (Vector Laboratories, Inc.) according to the manufacturer's protocol and visualized with 3,3'-diaminobenzidine in a buffered substrate (Nichirei Biosciences, Inc.). To determine the relative intensity on the cell surface with PD-L1 staining, the average pixel intensity was determined from at least 20 randomly selected cells using ImageJ $1.51 \mathrm{~J} 8$.

miR target site prediction. Putative miRs targeting the 3'untranslated region (UTR) sequences of CMTM4 and CMTM6 transcripts were determined using TargetScan 7.2 software (14).

Statistical analyses. Statistically significant differences between two groups were identified using Student's t-test when the variances were equal and Welch's t-test when the variances were unequal. When comparing multiple groups, ANOVA and Bonferroni post hoc test were used to determine the statistical significances. P-values $<0.05$ were considered to indicate statistical significance.

\section{Results}

Establishment of CDR1-AS-expressing SW620 colon cancer cells. To establish stably-CDR1-AS-expressing colon cancer cells, laccase cassettes were used to construct lentiviruses containing CDR1-AS sequences, which efficiently produced circular RNAs because a pair of inverted DNAREP1_DM family transposons were located very close to the circularizing exon and these repeats are highly complementary to each other (15) (Fig. 1A). The viruses were infected into SW620 cells, a colon cancer cell line with relatively high miR7 expression (16). To confirm the circularity of the expressed CDR1-AS RNA, the expression after RNase R treatment, which digests all linear RNAs but does not digest lariat or circular RNA structures, was examined by Northern blotting with a probe against the back-splice junction of the CDR1-AS RNA (Fig. 1B). CDR1-AS was almost the expected length (1,480 nucleotides) in agarose gel electrophoresis, and the bands were visible even after RNase R treatment, whereas the bands corresponding to $\beta$-actin completely disappeared, indicating that CDR1-AS forms a circular RNA resistant to RNase R treatment (Fig. 1B). The high intensity levels of bands from the CDR1-AS-expressing constructs (from 2,000 to 4,000 nucleotides) disappeared after RNase R treatment. It was speculated that these bands corresponded to incompletely circularized transcripts and/or nicked circular RNAs. As RNase R is a 3'-5' exoribonuclease that digests RNAs with 3'ends, completely circularized CDR1-AS transcripts were unaffected and remained even after RNase R treatment. RT-PCR determined that CDR1-AS RNA was expressed at levels 300 times higher than those in the non-expressing control cells, and did not significantly diminish even after RNase R treatment (Fig. 1C). Consistent with a previous study (6), CDR1-AS expression restored miR7 function as determined by a reporter assay, which was reversed by the forced expression of miR7-mimic LNA oligonucleotides (Fig. 1D). Although these results confirmed that CDR1-AS acted as an inhibitor of miR7 function in our system, endogenous miR7 levels were not prominent in colon cancer cells (8).

CDR1-AS does not affect the growth or invasiveness of colon cancer cells. Since CDR1-AS expression is closely linked with poor prognosis in various cancers, including colon carcinoma $(5,17)$, the effects of CDR1-AS on the growth and invasiveness of SW620 cells were examined. The growth of SW620 cells in both 2D and 3D cultures was examined since a $3 \mathrm{D}$ culture may render different results from conventional 2D cultures; however, there was no significant growth advantage observed in CDR1-AS-expressing SW620 cells (Fig. 2A and B). Furthermore, a Transwell invasion assay did not reveal any increased invasiveness of CDR1-AS-expressing SW620 cells (Fig. 2C). Angiogenesis was also examined using supernatant from CDR1-AS-expressing SW620 cells to determine whether they promote angiogenesis. However, there were no significant differences in the length and branch counts in in vitro angiogenesis between the supernatants from the control and the CDR1-AS-expressing cells (Fig. 2D). These 

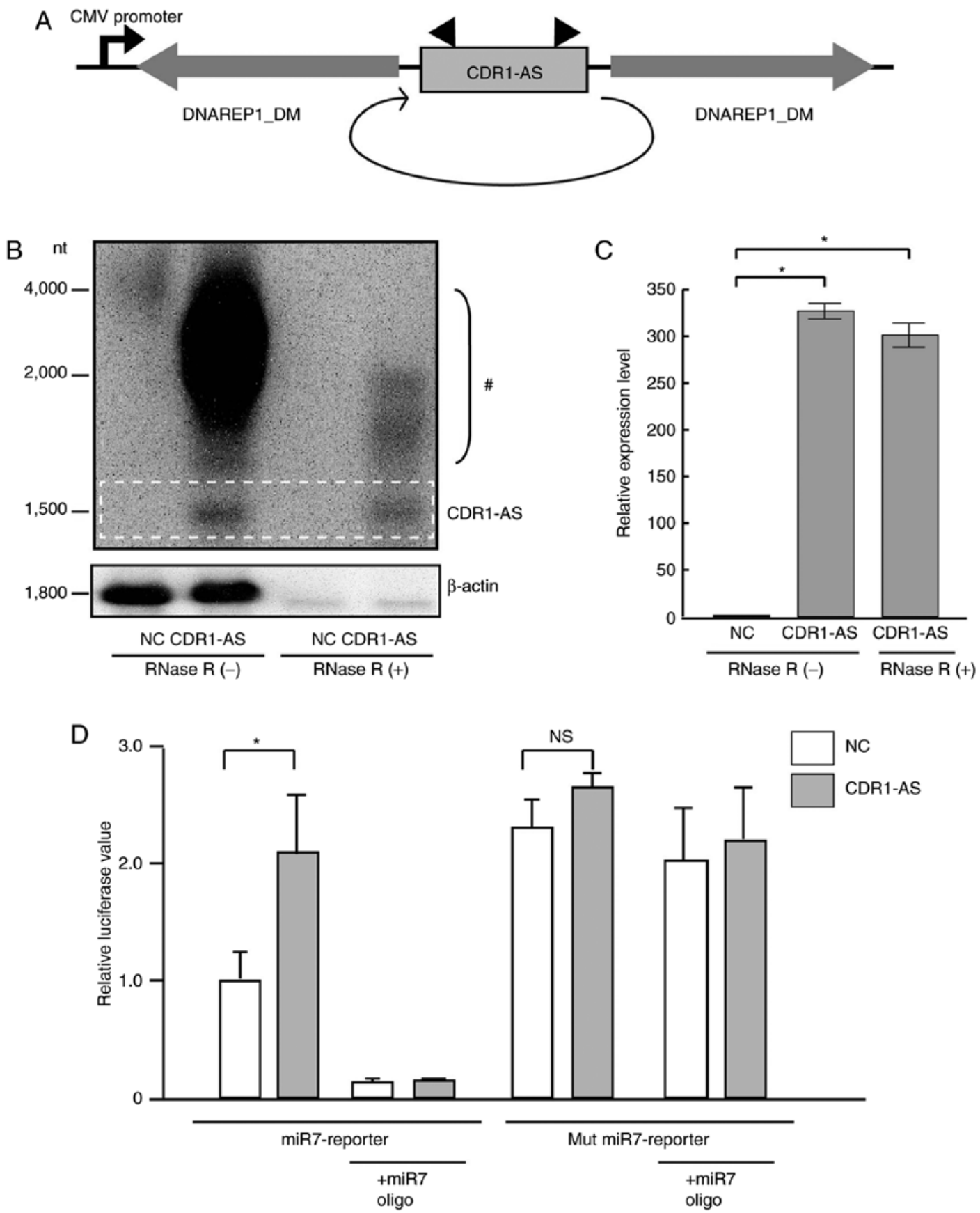

Figure 1. Establishment of CDR1-AS-expressing SW620 cells. (A) Construction of CDR1-AS RNA-expressing lentiviruses. CDR1-AS RNA is expressed under the CMV promoter. A pair of inverted transposable Drosophila melanogaster gene (DNAREP1_DM) sequences outside of the CDR1-AS sequence enhance the circularization of the CDR1-AS gene. Arrowheads indicate the primer positions used for RT-PCR to quantitate CDR1-AS RNA levels. (B) Northern blotting for CDR1-AS. RNAs extracted from control and CDR1-AS-expressing cells were subjected to Northern blotting. RNase R treatment was used to digest linear RNAs. $\beta$-actin mRNA was visualized to confirm the equal loading of RNA and the successful treatment with RNase R. A representative image of three independent experiments is presented. The dashed white box indicates the position of CDR1-AS. The (\#) indicates the linear CDR1-AS transcripts and CDR1-AS with possible higher structures. nt, nucleotides. (C) Increased CDR1-AS levels in control and CDR1-AS-expressing cells were confirmed by qRT-PCR. RNAs extracted from CDR1-AS-expressing SW620 cells, followed by treatment with RNase R, were also included. Data represent the mean \pm SD of triplicate experiments. ${ }^{*} \mathrm{P}<0.05$. (D) miR-7 function was impaired in CDR1-AS-expressing SW620 cells. A reporter construct to assess miR7 function was transiently transfected into negative control and CDR1AS-expressing SW620 cells with and without miR7 mimic LNA oligonucleotides. Results with a reporter containing mutated miR7 response sequences are also presented as controls. Data represent the mean $\pm \mathrm{SD}$ of triplicate experiments. ${ }^{*} \mathrm{P}<0.05$. CDR1-AS, cerebellar degeneration-related protein 1 transcript.

results appear to exclude the possibility that angiogenetic factors are produced by CDR1-AS expression and suggest that, although CDR1-AS expression in colon cancer cells is clinically linked with poor prognosis, cell growth, invasiveness, and angiogenesis are unaffected.
CMTM4 and CMTM6 IRNA levels are increased in CDR1-AS-expressing SW620 cells. To gain insight into the biological causes of the poor prognosis of colon cancers with CDR1-AS expression, changes in transcript expression levels in CDR1-AS-expressing SW620 cells (the cDNA microarray 
A

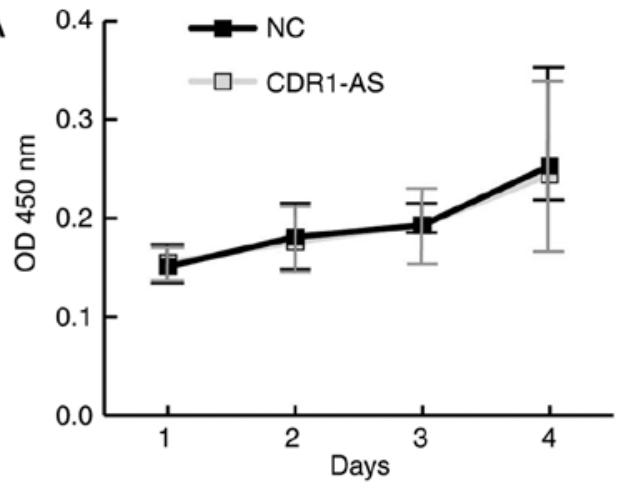

B $5.0 \times 10^{6}$

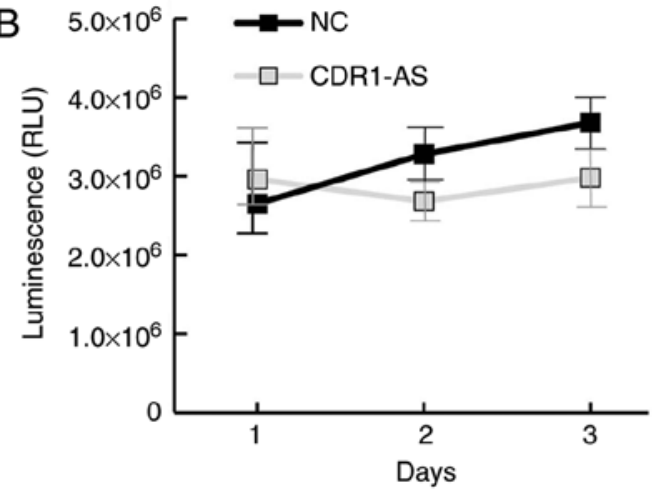

D

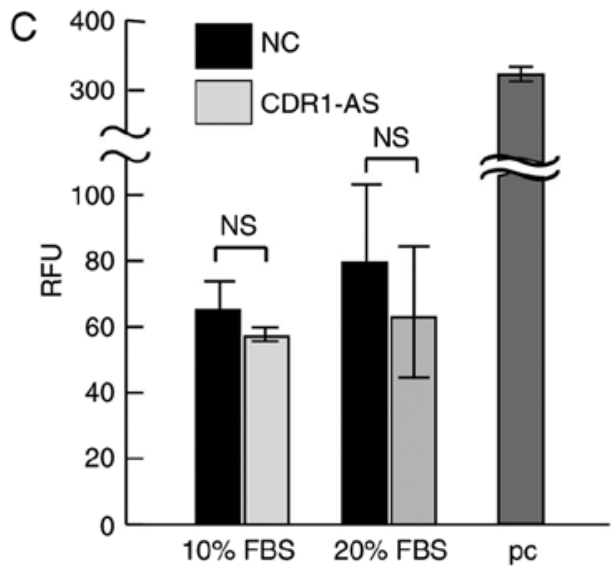

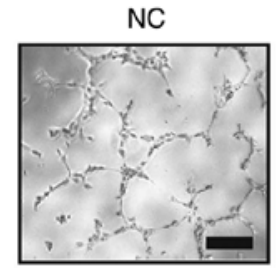
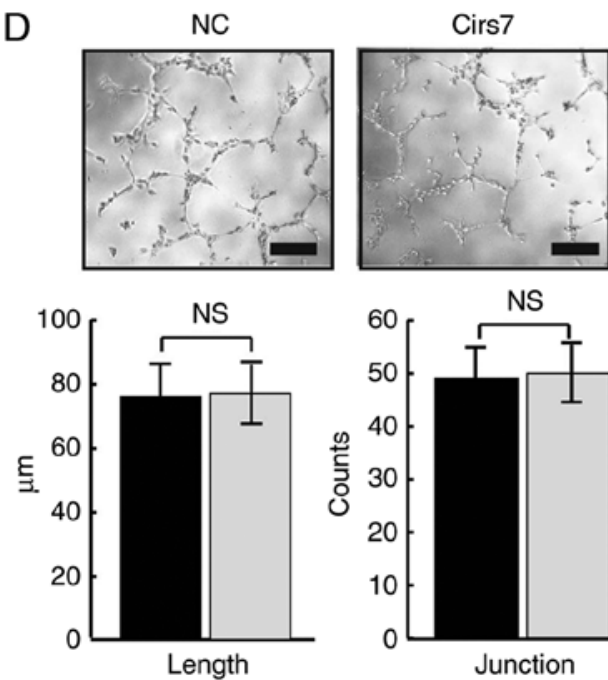

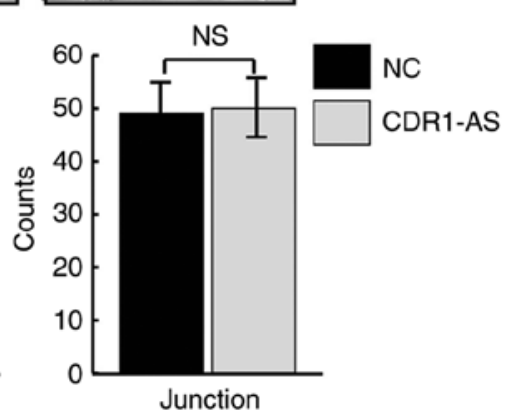

Figure 2. CDR1-AS RNA expression does not affect growth, invasion, or angiogenesis. (A and B) Cell growth rate was compared between the negative control and CDR1-AS-expressing SW620 cells in (A) 2D and (B) 3D-culture conditions. Data represent the mean \pm SD of triplicate experiments. Cell growth was determined using the OD values at $450 \mathrm{~nm}$ in 2D culture and the RLU in 3D culture. (C) The cell invasion rate was compared between the control and CDR1-AS-expressing SW620 cells using invasion chambers covered with a layer of dried basement membrane matrix solution. FBS (10 and 20\%) was used as a chemoattractant. HT1080 cells whose invasion was induced by 10\% FBS were included as a positive control (pc). Data represent the mean \pm SD of triplicate experiments. (D) The angiogenesis rate was compared in endothelial tube formation between the culture supernatant of the negative control and CDR1-AS-expressing SW620 cells. The tube length and the number of junctions were assessed. The top panels show representative images of angiogenesis. Bar, $300 \mu \mathrm{m}$. Data represent the mean \pm SD of triplicate experiments in the lower panels. CDR1-AS, cerebellar degeneration-related protein 1 transcript; RLU, relative light unit; RFU, relative fluorescence units; ns, not significant.

results were deposited in GEO; accession no. GSE125687) were assessed. The simultaneous increases in CMTM4 and CMTM6 transcript levels were noteworthy since these genes were recently reported as stabilizers of PD-L1 protein at the cell surface $(18,19)$. The PD-L1 ligand plays a crucial role in suppressing tumor-specific T-cell responses, and its upregulation on the surface of cancer cells is linked to enhanced inhibition of $\mathrm{T}$ cells and thus, poor prognosis (20). The upregulation of CMTM4 and CMTM6 transcript levels using RT-PCR was confirmed (Fig. 3A). Accordingly, cell surface PD-L1 levels, which were expressed only in a subpopulation, were upregulated in CDR1-AS-expressing SW620 cells (Fig. 3B and C), although PD-L1 mRNA levels were unchanged (Fig. 3A). The increased expression of PD-L1 on the surface of CDR1-AS-expressing cells was also detected in xenograft models established using SW620 cells (Fig. 3D and E), although tumour growth/size was not analysed in the present study in the presence/absence of CDR1AS expression due to inconsistencies in the growth rates rendering the appropriate analysis difficult. Similarly, in Caco2 cells, another colon cancer cell line, forced expression of CDR1-AS RNA (Fig. S1A) induced significantly increased expression of CMTM transcripts (Fig. S1B) and PD-L1 protein on the cell surface (Fig. S1C and D). These results inidcated that CDR1-AS expression leads to an increase in CMTM4 and CMTM6 expression levels, resulting in an increase in cell surface PD-L1 levels.

Increased cell surface PD-L1 expression in CDR1-ASexpressing cells is not dependent on miR7 function. Since CDR1-AS functionally antagonizes miR7 due to sequence similarities (Fig. 1D) (6,7), miR7 mimic LNA oligonucleotides were ectopically expressed in CDR1-AS-expressing cells to examine the involvement of deregulated miR7 function in the upregulated cell surface PD-L1 expression levels. However, the increased CMTM4 and CMTM6 transcript levels (Fig. 3F) and the cell surface PD-L1 protein levels (Fig. 3G), which were expressed in a subpopulation of the cells, were not reduced by forced expression of miR7 mimic LNA oligonucleotides. These results were consistent with the fact that the 3'UTRs 

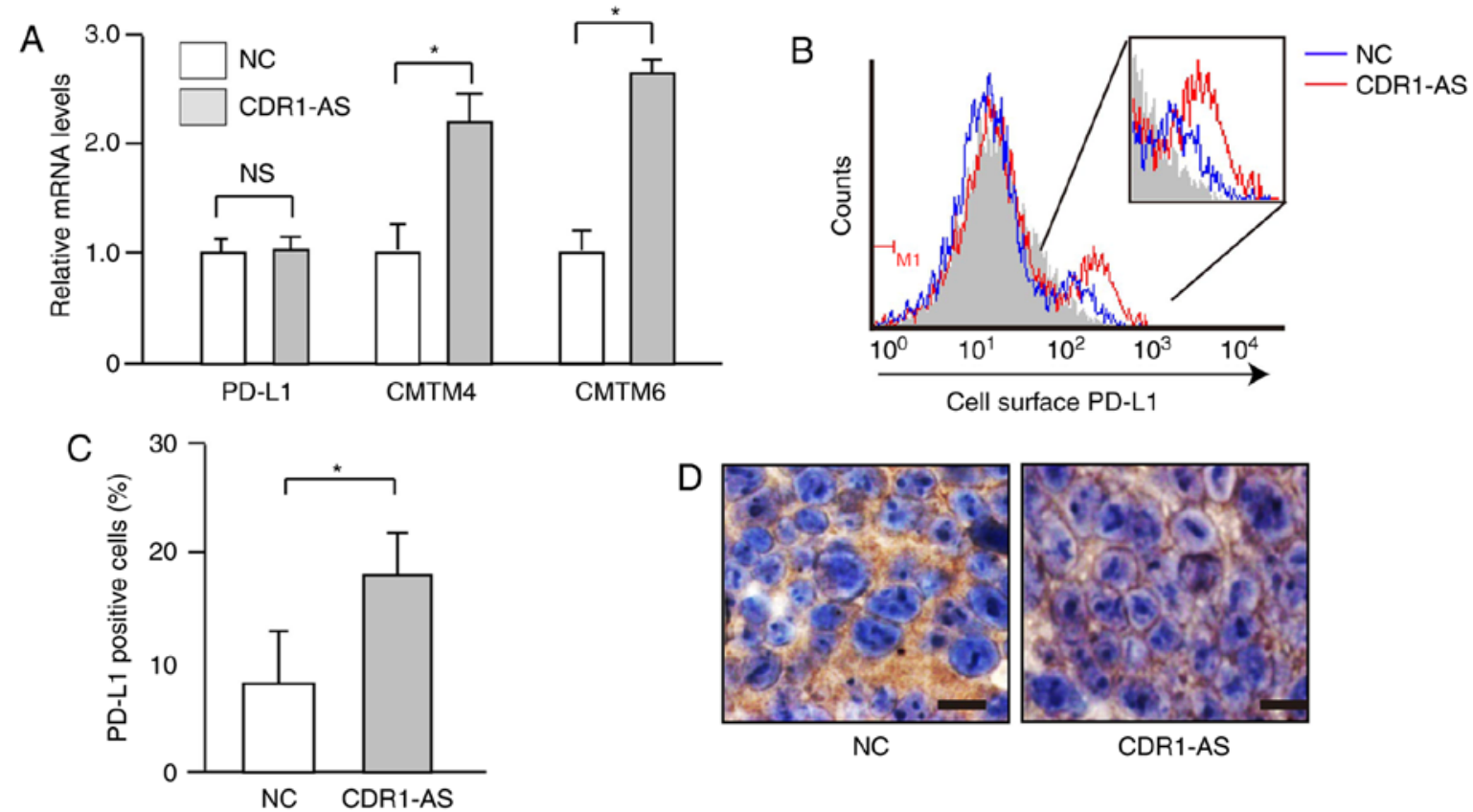

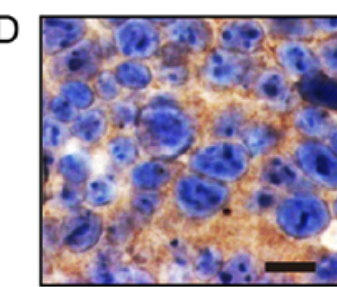

$\mathrm{NC}$

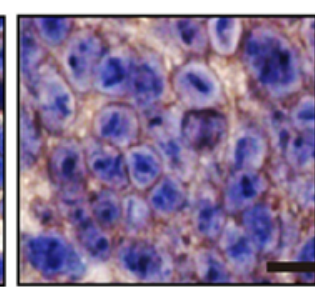

CDR1-AS
E

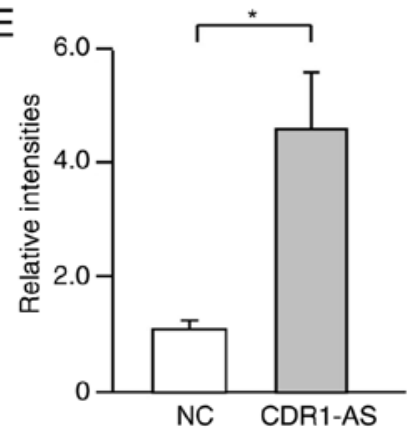

G

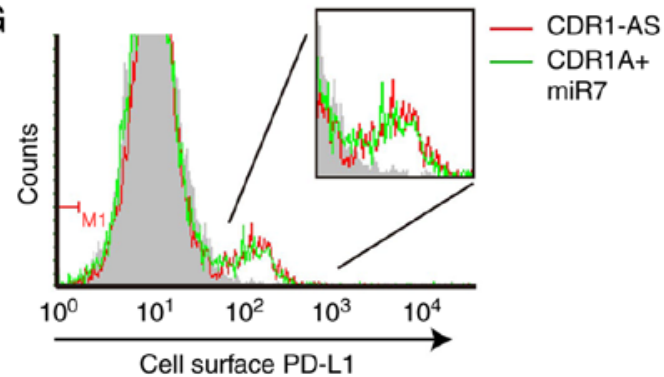

$\mathrm{F}$

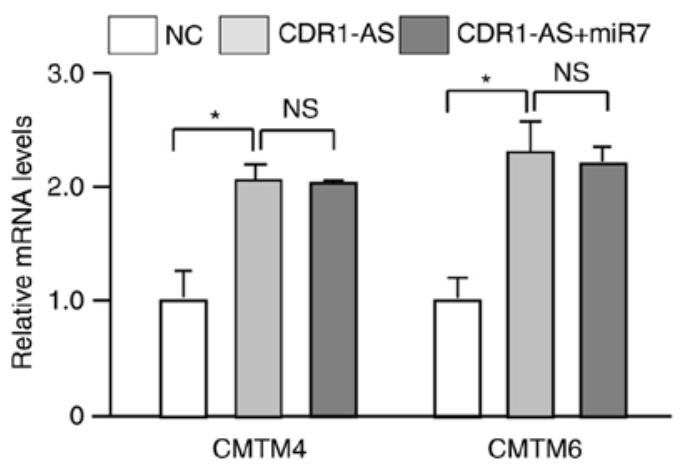

$\mathrm{H}$

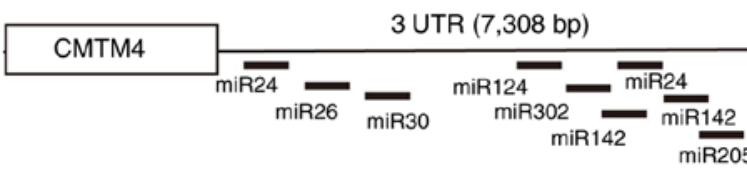

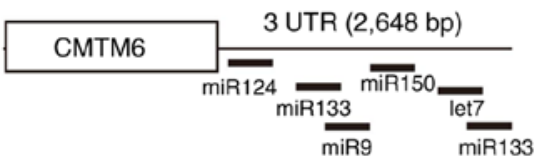

Figure 3. Cell surface PD-L1 protein levels were increased in CDR1-AS-expressing cells. (A) qRT-PCR indicated the expression levels of the indicated transcripts in control and CDR1-AS-expressing SW620 cells. Data represent the mean \pm SD of triplicate experiments. "P<0.05. (B) FACS analyses revealed an increase in cell surface PDL1 protein expression levels in a subpopulation of CDR1-AS-expressing cells. The inset indicates the population subset expressing PD-L1 protein at the cell surface. A representative image of three independent experiments is presented. (C) The percentage of PD-L1-positive cells relative to the negative control using the isotype IgG was determined by FACS analyses. Data represent the mean $\pm \mathrm{SD}$ of triplicate experiments. "P<0.05. (D) Immunohistochemistry revealed increased cell surface PD-L1 protein levels (brown) in xenograft tissues established using CDR1-AS-expressing cells compared with those using control cells. A representative image of three independent experiments is shown. Bar, $20 \mu \mathrm{m}$. (E) The intensity of cell surface staining with PD-L1, of at least 20 randomly selected cells in each immunohistochemical experiment, was determined by ImageJ analysis; the relative intensities were calculated. Data represent the mean $\pm \mathrm{SD}$ of two xenografts from two mice in each group. " $\mathrm{P}<0.05$. (F) CMTM6 RNA expression levels were not reduced by the forced expression of miR7 mimic LNA oligonucleotides in CDR1-AS-expressing SW620 cells, as determined by RT-PCR. Data represent the mean \pm SD of triplicate experiments. ${ }^{*} \mathrm{P}<0.05$. (G) Cell surface PD-L1 protein levels were unaffected by the forced expression of miR7 mimic LNA oligonucleotides in CDR1-AS-expressing SW620 cells. The inset indicates the population subset expressing cell surface PD-L1 protein. A representative image of three independent experiments is shown. (H) Putative miR target sequences in the 3'UTRs of CMTM4 and CMTM6 transcripts, identified using TargetScan 7.2 software. CDR1-AS, cerebellar degeneration-related protein 1 transcript; ns, not significant.

of CMTM4 and CMTM6 do not share sequence similarities with miR7 (Fig. 3H). Thus, the upregulated expression levels of CMTM4 and CMTM6 in CDR1-AS-expressing cells do not appear to be dependent on impaired miR7 function, but likely depend on other undefined factors induced by CDR1-AS expression. 


\section{Discussion}

In the present study, it was revealed that the expression of circular RNA CDR1-AS in colon cancer cells induces the upregulation of CMTM6 expression levels, which may be linked with increased PD-L1 protein expression on the cell surface. This may underlie the poor prognosis in colon cancer cases with CDR1-AS expression.

CDR1-AS is frequently overexpressed in colon cancer tissues compared with normal tissues. High CDR1-AS expression levels have been revealed to be correlated with poor survival in patients $(5,21)$. In the present study, unexpectedly, cell growth, invasion ability, and vascular growth did not increase according to CDR1-AS expression, although these are often reported as results of impaired miR7 function by CDR1-AS (22). This may be due to differences in the balance between miR7 expression levels and the expression levels of CDR1-AS in the cells. Instead, it was revealed that cell surface expression levels of PD-L1 protein were upregulated in CDR1-AS-expressing colon cancer cells. Sicne PD-L1 expression on tumor cells is associated with poor prognosis in patients with colorectal cancer, possibly through T-cell inhibition by cell surface PD-L1 expression in cancer cells (20), this may account for the poor prognosis of colon cancer patients with high CDR1-AS expression levels.

CMTM6/4 were recently identified as regulators of PD-L1 stability at the cell surface $(18,19)$. Although CDR1-AS is recognized to antagonize miR7 function (6), the 3'UTR sequences of CMTM6 and CMTM4 do not contain any similar sequences complementary to miR7. Additionally, overexpression of miR7 in CDR1-AS-expressing cells did not decrease the expression levels of CMTM6 or CMTM4 mRNA. Although the mechanisms underlying the regulation of the expression of CMTM6 or CMTM4 remain unknown, the present results indicated that the upregulation of CMTM6 and CMTM4 by CDR1-AS expression may indirectly affect expression by modulating the function or expression of transcription factors required for CMTM6 and CMTM4 expression.

To further confirm the involvement of CMTM6 and CMTM4 in the changes of PD-L1 expression levels by CDR1-AS, blocking experiments of CMTM6 and CMTM4 were attempted in CDR1-AS-expressing cells, however, solid data could not be obtained because it was difficult to control the knockdown levels of CMTM4 and CMTM6. Thus, these data were omitted from this study. Several studies have reported that CMTM4 and CMTM6 are involved in the cell surface expression of PD-LI $(18,19)$. The decreased PD-L1 expression in CMTM4 and CMTM6-knockdown cells was also observed, which suggested the involvement of these proteins in PD-L1 expression.

Since CDR1-AS expression is closely linked with poor prognosis in various cancers $(5,22)$, our findings may be generally applied to other cancers in addition to colon cancer. Although the mechanism by which CDR1-AS expression is upregulated in some cancers is unknown, interventional methods against CDR1-AS as a non-coding RNA may enhance the effectiveness of current PD-L1-PD1 blocking therapies. In summary, a link between the expression of a circular RNA and its onco-immunological function was identified, which explains why some cancer patients with high CDR1-AS expression have a poor prognosis.

\section{Acknowledgements}

Not applicable.

\section{Funding}

The present study was supported by Grants-in-Aid from the Ministry of Education, Culture, Sports, Science and Technology, Japan (nos. 16H05149, 16KT0109 and 17K15923) (to MO and TK), by the Grant-in-Aid for Scientific Research on Innovative Areas (no. 18H05024 to MO), by The Yasuda Medical Foundation (to MO), and by the Project for Cancer Research And Therapeutic Evolution (P-CREATE) from Japan Agency for Medical Research and Development (AMED) (to MO, no. JP18cm0106602).

\section{Availability of data and materials}

The datasets used during the present study are available from the corresponding author upon reasonable request.

\section{Authors' contributions}

ET and MO planned the research and wrote the manuscript. ET, YM, TK, TSe, TI, KF, NO, KS, MY, and TSu performed majority of the experiments. RI and MO analyzed the data. KK supervised the entire project and wrote the manuscript. All authors read and approved the final version to be published and agree to be accountable for all aspects of the work in ensuring that questions related to the accuracy or integrity of any part of the work are appropriately investigated and resolved.

\section{Ethics approval and consent to participate}

Experimental protocols were approved by the Ethics Committee for Animal Experimentation at the University of Tokyo (Approval no. P18-017) and conducted in accordance with the Guidelines for the Care and Use of Laboratory Animals of the Department of Medicine, University of Tokyo.

\section{Patient consent for publication}

Not applicable.

\section{Competing interests}

The authors declare that they have no competing interests.

\section{References}

1. Tay Y, Rinn J and Pandolfi PP: The multilayered complexity of ceRNA crosstalk and competition. Nature 505: 344-352, 2014.

2. Ebbesen KK, Hansen TB and Kjems J: Insights into circular RNA biology. RNA Biol 14: 1035-1045, 2017.

3. Kristensen LS, Hansen TB, Ven $\varnothing$ MT and Kjems J: Circular RNAs in cancer: Opportunities and challenges in the field. Oncogene 37: 555-565, 2018.

4. Arnold M, Sierra MS, Laversanne M, Soerjomataram I, Jemal A and Bray F: Global patterns and trends in colorectal cancer incidence and mortality. Gut 66: 683-691, 2017. 
5. Weng W, Wei Q, Toden S, Yoshida K, Nagasaka T, Fujiwara T, Cai S, Qin H, Ma Y and Goel A: Circular RNA ciRS-7-A promising prognostic biomarker and a potential therapeutic target in colorectal cancer. Clin Cancer Res 23: 3918-3928, 2017

6. Hansen TB, Jensen TI, Clausen BH, Bramsen JB, Finsen B, Damgaard CK and Kjems J: Natural RNA circles function as efficient microRNA sponges. Nature 495: 384-388, 2013.

7. Memczak S, Jens M, Elefsinioti A, Torti F, Krueger J, Rybak A, Maier L, Mackowiak SD, Gregersen LH, Munschauer M, et al: Circular RNAs are a large class of animal RNAs with regulatory potency. Nature 495: 333-338, 2013.

8. Horsham JL, Ganda C, Kalinowski FC, Brown RA, Epis MR and Leedman PJ: MicroRNA-7: A miRNA with expanding roles in development and disease. Int J Biochem Cell Biol 69: 215-224, 2015.

9. Kishikawa T, Otsuka M, Yoshikawa T, Ohno M, Yamamoto K, Yamamoto N, Kotani A and Koike K: Quantitation of circulating satellite RNAs in pancreatic cancer patients. JCI Insight 1 : e86646, 2016.

10. Yamagami M, Otsuka M, Kishikawa T, Sekiba K, Seimiya T, Tanaka E, Suzuki T, Ishibashi R, Ohno M,and Koike K: ISGF3 with reduced phosphorylation is associated with constitutive expression of interferon-induced genes in aging cells. NPJ Aging Mech Dis 4: 11, 2018.

11. Livak KJ and Schmittgen TD: Analysis of relative gene expression data using real-time quantitative PCR and the 2(-Delta Delta C(T)) method. Methods 25: 402-408, 2001.

12. Sekiba K, Yamagami M, Otsuka M, Suzuki T, Kishikawa T, Ishibashi R, Ohno M, Sato M and Koike K: Transcriptional activation of the MICA gene with an engineered CRISPR-Cas9 system. Biochem Biophys Res Commun 486: 521-525, 2017.

13. Yoshikawa T, Wu J, Otsuka M, Kishikawa T, Suzuki N, Takata A Ohno M, Ishibashi R, Yamagami M, Nakagawa $\mathrm{R}$, et al: Repression of MicroRNA function mediates inflammation-associated colon tumorigenesis. Gastroenterology 152: 631-643, 2017.
14. Agarwal V, Bell GW, Nam JW and Bartel DP: Predicting effective microRNA target sites in mammalian mRNAs. Elife 4: 2015.

15. Kramer MC, Liang D, Tatomer DC, Gold B, March ZM, Cherry S and Wilusz JE: Combinatorial control of Drosophila circular RNA expression by intronic repeats, hnRNPs, and SR proteins. Genes Dev 29: 2168-2182, 2015.

16. Zhang N, Li X, Wu CW, Cai M, Mok MT, Wang H, Chen J, Ng SS, Chen M, Sung JJ and Yu J: microRNA-7 is a novel inhibitor of YY1 contributing to colorectal tumorigenesis. Oncogene 32: 5078-5088, 2013

17. Peng L, Yuan XQ and Li GC: The emerging landscape of circular RNA ciRS-7 in cancer (Review). Oncol Rep 33: 2669-2674, 2015.

18. Mezzadra R, Sun C, Jae LT, Gomez-Eerland R, de Vries E, Wu W, Logtenberg MEW, Slagter M, Rozeman EA, Hofland I, et al: Identification of CMTM6 and CMTM4 as PD-L1 protein regulators. Nature 549: 106-110, 2017.

19. Burr ML, Sparbier CE, Chan YC, Williamson JC, Woods K, Beavis PA, Lam EYN, Henderson MA, Bell CC, Stolzenburg S, et al: CMTM6 maintains the expression of PD-L1 and regulates anti-tumour immunity. Nature 549: 101-105, 2017.

20. Chen DS, Irving BA and Hodi FS: Molecular pathways: Next-generation immunotherapy-inhibiting programmed death-ligand 1 and programmed death-1. Clin Cancer Res 18: 6580-6587, 2012

21. Tang W, Ji M, He G, Yang L, Niu Z, Jian M, Wei Y, Ren L and $\mathrm{Xu}$ J: Silencing CDR 1as inhibits colorectal cancer progression through regulating microRNA-7. Onco Targets Ther 10: 2045-2056, 2017.

22. Zhang J, Hu H, Zhao Y and Zhao Y: CDRlas is overexpressed in laryngeal squamous cell carcinoma to promote the tumour's progression via miR-7 signals. Cell Prolif 51: e12521,2018. 